\title{
Prevalence of major levator abnormalities in symptomatic patients with an underactive pelvic floor contraction
}

\author{
Anneke B. Steensma • Maja L. Konstantinovic • \\ Curt W. Burger • Dirk de Ridder • Dirk Timmerman • \\ Jan Deprest
}

Received: 15 April 2009 /Accepted: 11 January 2010 /Published online: 4 March 2010

(C) The Author(s) 2010. This article is published with open access at Springerlink.com

\begin{abstract}
Introduction and hypothesis Major levator ani abnormalities (LAA) may lead to abnormal pelvic floor muscle contraction (pfmC) and secondarily to stress urinary incontinence (SUI), prolapse, or fecal incontinence (FI).

Methods A retrospective observational study included 352 symptomatic patients to determine prevalence of LAA in underactive $\mathrm{pfmC}$ and the relationship with symptoms. On 2D/3D transperineal ultrasound, $\mathrm{PfmC}$ was subjectively assessed as underactive $(\mathrm{UpfmC})$ or normal $(\mathrm{NpfmC})$ and quantified. LAA, defined as a complete avulsion of the pubic bone, was analyzed using tomographic ultrasound imaging.

Results LAA were found in $53.8 \%$ of women with UpfmC versus $16.1 \%$ in $\mathrm{NpfmC}(P<0.001)$. Patients with UpfmC were less likely to reduce hiatal area on pfmC (mean 7\% reduction vs $25 \%$ in $\mathrm{NpfmC}(P<0.001))$. An UpfmC was associated with FI $(P=0.002)$, not with SUI or prolapse of the anterior and central compartment.

Conclusion An underactive pfmC is associated with increased prevalence of LAA and FI.
\end{abstract}

\footnotetext{
A. B. Steensma $\cdot$ C. W. Burger

Department of Gynaecology and Gynaecooncology,

Erasmus Medical Center,

Rotterdam, The Netherlands Department of Urogynaecology, UZ Leuven,

Campus Gasthuisberg,

Leuven, Belgium

\author{
A. B. Steensma $(\bowtie)$ \\ Erasmus Medical Center, Rotterdam, \\ Room He 124's, Gravendijkwal 230, \\ 3015 CE Rotterdam, The Netherlands
}

Department of Obstetrics and Gynaecology,

e-mail: a.b.steensma@erasmusmc.nl
}

M. L. Konstantinovic · D. de Ridder · D. Timmerman · J. Deprest
Keywords Fecal incontinence - Levator ani abnormalities · Pelvic floor muscle contraction - Stress urinary incontinence . Prolapse $\cdot$ Transperineal ultrasound

$\begin{array}{ll}\text { Abbreviations } \\ \text { SUI } & \text { Stress urinary incontinence } \\ \text { TUI } & \text { Tomographic ultrasound imaging } \\ \text { AP } & \text { Antero-posterior } \\ \text { FI } & \text { Fecal incontinence } \\ \text { 3D } & \text { 3-dimensional } \\ \text { 2D } & \text { 2-dimensional } \\ \text { ICS POP-Q } & \text { International Continence Society } \\ & \text { Pelvic-Organ-Prolapse Quantification } \\ \text { pfmC } & \text { Pelvic floor muscle contraction } \\ \text { UpfmC } & \text { Underactive pelvic floor muscle contraction } \\ \text { NpfmC } & \text { Normal pelvic floor muscle contraction } \\ \text { PFMF } & \text { Pelvic floor muscle function } \\ \text { POP } & \text { Pelvic organ prolapse } \\ \text { LAA } & \text { Major levator ani abnormalities }\end{array}$

\section{Introduction}

The levator ani muscle complex is attached to the internal surface of the true pelvis. It is divided into three parts according to their attachments and pelvic viscera to which they are related, i.e., the ileococcygeal, pubococcygeal, and puborectal muscle. Appropriate contraction and relaxation result in optimal pelvic floor support and function of the levator ani. Contractility of the pelvic floor may play an important role in sustaining continence and or preventing pelvic organ prolapse (POP).

Clinical assessment of pelvic floor muscle function (PFMF) is typically performed, subjectively, by vaginal 
digital palpation, however, with poor repeatability [1]. In 2005, Messelink et al. recommended to quantify contractions by the modified Oxford scale as either absent, weak, normal, or strong [2]. Observation and measurement of normal or abnormal PFMF by vaginal palpation is often difficult due to the anatomical location of the levator ani. The levator ani can be visualized with magnetic resonance imaging (MRI) as well as three-dimensional (3D) transperineal ultrasound [3-5]. While MRI is invasive, expensive, and not widely available, 3D transperineal ultrasound offers equal resolution for the inferior components of the levator ani, is less expensive, and well-tolerated by the patient [6]. Both Ultrasound and MRI can be used for objective measurements of analyzing PFMF aspects, such as closure or reduction of the dimensions of the levator hiatus on pelvic floor contraction [7]. Furthermore, pelvic floor ultrasound can quantify PFMF, but data on normal or abnormal PFMF are lacking. With increasing experience in both dynamic imaging methods, new insights have been gained in the function and anatomy of the PFMF in patients with pelvic floor disorders. Apart from a functional estimation, little is known about the relationship between levator ani structure (morphological and functional integrity), the quality of its contraction, and the, eventually, relationship with symptoms. It is thought that damage to the levator ani muscle can lead to poor function and secondarily, can lead to symptoms such as urine incontinence, prolapse, and fecal incontinence (FI) [8-11].

To date, there is no information on the relationship between the integrity of the pelvic floor and success of treatment [12]. Pelvic floor re-education and muscle training is a well-accepted treatment for pelvic floor symptoms, especially for stress urinary incontinence (SUI) [13]. In a number of patients, conservative treatment fails, which has not been related to the underlying anatomic condition. We designed the present study to quantify subjective analysis of normal and abnormal PFMF on two-dimensional (2D)/3D pelvic floor ultrasound and to investigate an eventual relationship between anatomical and functional abnormalities of the levator complex, as well as their relation to pelvic floor disorder symptoms.

\section{Materials and methods}

Between August 2006 and April 2007, all patients who were referred to two tertiary pelvic floor clinics because of pelvic floor disorder symptoms, such as SUI, prolapse, and/ or FI, were included in this study. They underwent a standardized interview, clinical examination according to the International Continence Society Pelvic-Organ-Prolapse Quantification (ICS POP-Q) staging. SUI was defined according the ICS terminology as involuntary leakage on effort or exertion or on sneezing or coughing [14] and considered to be of a relevant complaint only if there was more than once a week involuntary urine loss. FI was defined as involuntary loss of liquid or hard stool. Transperineal ultrasound was performed in the supine position and after voiding. 3D and four-dimensional (4D) volume datasets were acquired at rest, on pelvic floor contraction and on Valsalva maneuvre, using GE Kretz Voluson 730 Expert system and a RAB 4-8 MHZ probe as previously described by Dietz [15]. Off-line analysis was performed using the GE Kretz 4D view 5.0 software (GE Healthcare, Kretztechnik, Zipf, Austria) with the investigators blinded against clinical data.

The quality of pelvic floor muscle contractions was subjectively evaluated on the $2 \mathrm{D}$ cine loop volume with the best contraction assessed on transperineal ultrasound independently by the first two authors (A. B. S. and M. L. K.). The standardized ICS terminology for assessment of pelvic muscle contraction was used, scoring the contraction as absent, weak, normal, or strong $[2,13]$. We defined a poor PFMF as an "underactive pelvic floor contraction" (UpfmC) if there was evidence of an absent or weak pelvic floor contraction on ultrasonography,

Fig. 1 Antero-posterior measurements in $2 \mathrm{D}$ at the level of minimal hiatal dimension in rest position (left panel) and during contraction (right panel)

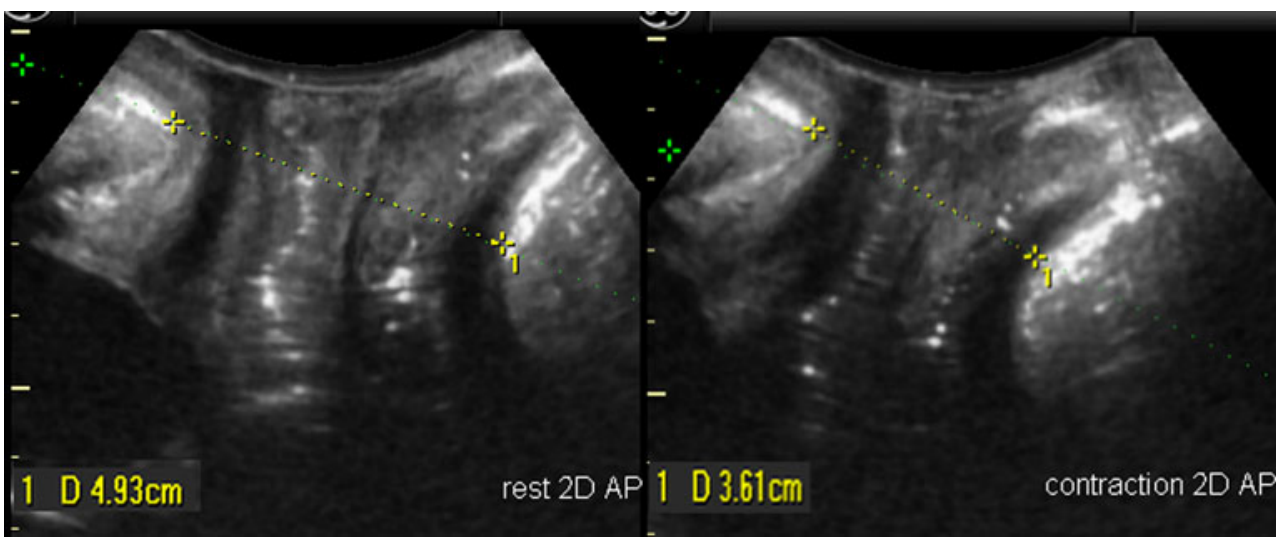


Fig. 2 Antero-posterior, transverse LR and hiatal area measurements in 3D at the level of the minimal hiatal dimension in rest position (left panel) and during contraction (right panel)

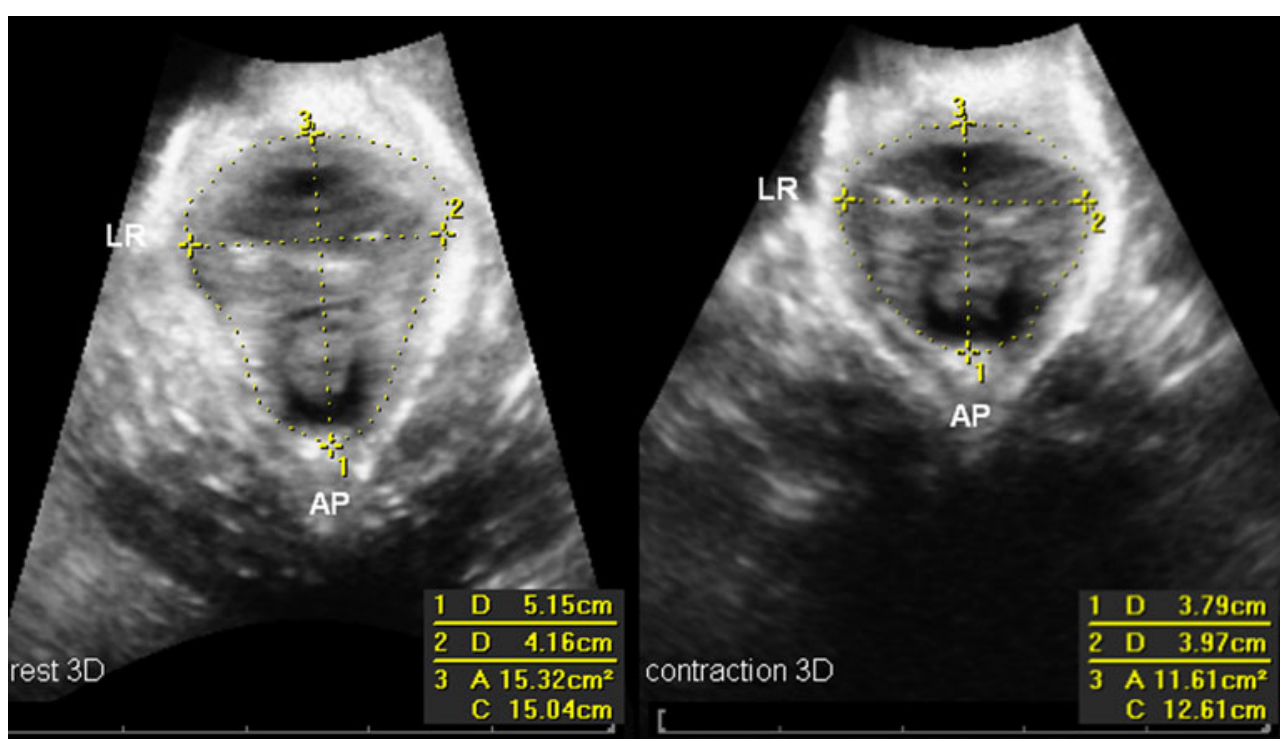

resulting in no or minimal changes in the reduction of the levator hiatus). A normal function $(\mathrm{NpfmC})$ of the pelvic floor was defined as if there was a normal or strong contraction.
All measurements were taken at the level of the minimal hiatal dimension, using the inferior margin of the symphysis pubis as reference point in $2 \mathrm{D}$ and $3 \mathrm{D}$ datasets as described by Dietz et al. $[15,16]$. Antero-posterior (AP)

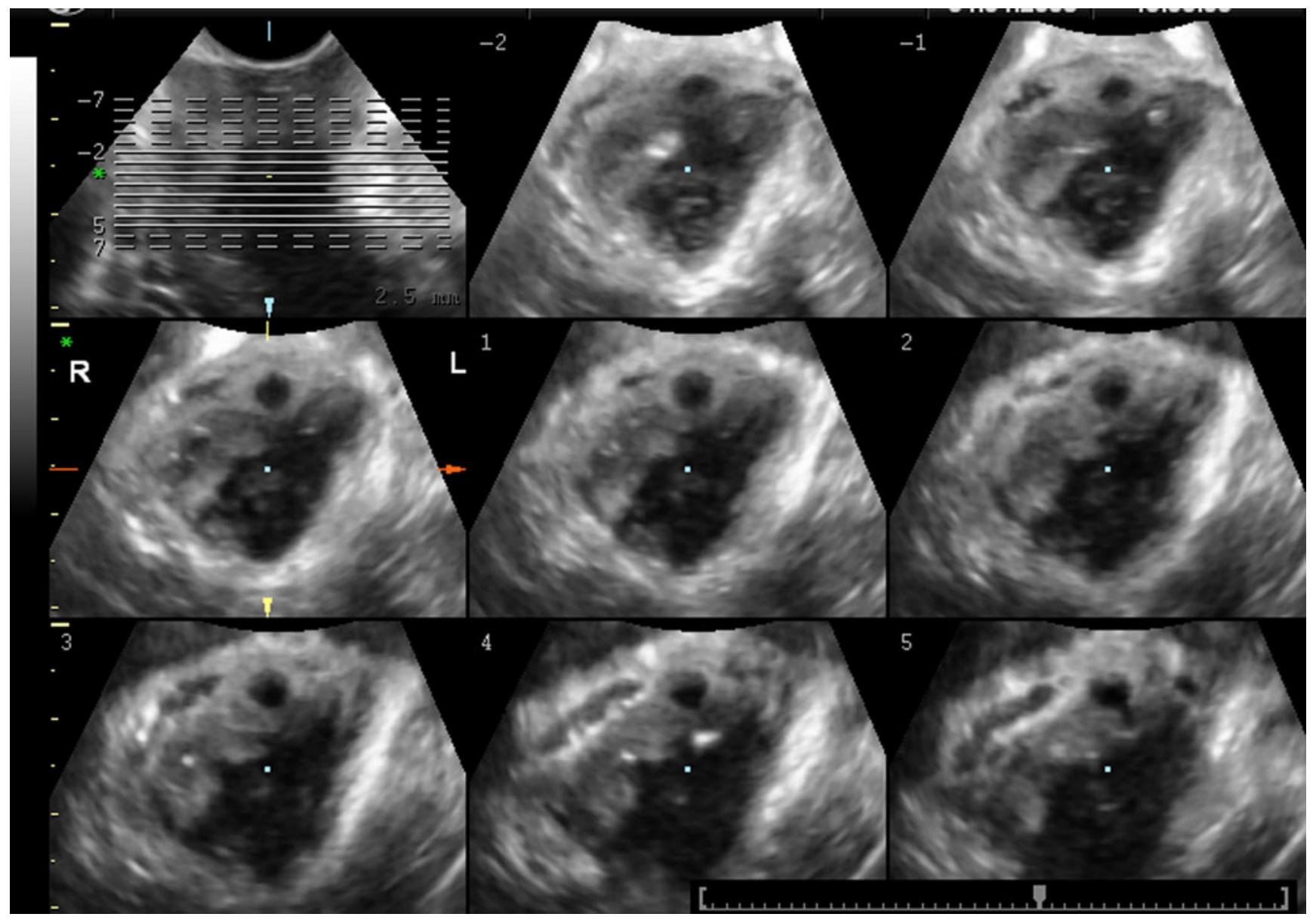

Fig. 3 Tomographic ultrasound imaging (TUI) imaging of the levator hiatus with an obvious unilateral defect on the right side (TUI score 8 for the right and 0 for left) 
Table 1 Subjective assessment of the pelvic floor muscle contraction $(\mathrm{pfmC})$ on transperineal ultrasound

\begin{tabular}{llrc}
\hline Contraction & Qualification & \multicolumn{1}{c}{$n$} & Percent, \% \\
\hline Underactive (UpfmC) & Absent & 45 & 13.4 \\
& Weak & 141 & 42.1 \\
\multirow{2}{*}{ Normal (NpfmC) } & Normal & 100 & 29.9 \\
& Strong & 49 & 14.6 \\
\hline
\end{tabular}

diameter and left-right transverse diameters as well as the hiatal area were measured at rest and during contraction (see Figs. 1 and 2). The percentage difference [(value A rest-value A contraction)/value A rest] for contraction was calculated as a measurement of PFMF.

A major levator ani abnormality (LAA), defect, or "avulsion" was defined as an obvious detachment of the levator ani muscle anteromedial from the pubic bone, either unilateral or bilateral $[4,5]$. For quantification of these levator defects, tomographic ultrasound imaging (TUI) was used [17]. A set of eight slices with an interslice interval of $2.5 \mathrm{~mm}$ was obtained, from $5.0 \mathrm{~mm}$ below to $12.5 \mathrm{~mm}$ above the hiatal plane, in a volume obtained on maximal levator contraction (see Fig. 3). A score of 0 was used if there were no defects on either side and a total score of 16 indicated a complete bilateral avulsion. A major levator ani defect was defined as a TUI score of eight for unilateral (left or right) and 16 for bilateral defects. All TUI evaluations were performed by one investigator (A. B. S.).

Both A. B. S. and M. L. K. performed off-line analysis of volume datasets of their own hospital, using the best contraction in the volume dataset of each patient. A testretest series was conducted in 50 volume datasets between the first two authors for a subjective evaluation of UpfmC and $\mathrm{NpfmC}$. A. B. S. had already gained extensive experience in analyzing pelvic floor volumes, and M. L. $\mathrm{K}$. was a trainee who did not have previous experience. Agreement was analyzed using Cohen's kappa.

Statistical analysis was performed using SPSS 15.0 (SPSS Inc., Chicago, USA). Pearson Chi-square and Student's $t$ correlations were used for comparison of normally distributed data and the Mann-Whitney $U$ test for nonparametric data. $P<0.05$ (two-sided) was considered statistically significant.

\section{Results}

During the observation period, 352 patients were included for this study. Complete datasets were available for 335 patients; $5 \%(n=17)$ of the patients were excluded due to incomplete clinical data and/or bad imaging quality. Two hundred eight patients attended the pelvic floor clinic in the Erasmus Medical Center (Rotterdam, The Netherlands) and 127 patients in the clinic in UZ Leuven, Campus Gasthuisberg (Leuven, Belgium). The mean age was 55.2 years ( 20 87 years) and median parity was $2.0(0-10)$. Their leading complaints were SUI in $34 \%(n=114)$, prolapse in $43.6 \%$ $(n=146)$, FI in $14.9 \%(n=50)$, and obstructed defecation in $9.3 \%(n=31)$ and/or a combination of complaints. Hysterectomy had previously been performed in $31.3 \%$ patients. Severe prolapse on clinical assessment (POP-Q stage two or more) was diagnosed in $43.6 \%(n=146)$, either from the anterior $(39.4 \% ; n=132)$, central $(19.1 \% ; n=64)$, and posterior $(29.3 \% ; n=98)$ compartment.

On assessing volume ultrasound data, an UpfmC was diagnosed in 186 patients $(55.5 \%)$, of whom $13.4 \%(n=45)$ had no visible (absent) pelvic floor contraction and $42.1 \%$ $(n=141)$ had a weak contraction on transperineal ultrasound (see Table 1). A test-retest series for qualitative assessment of PFMF on 3D ultrasound was conducted for 50 patients between the first two authors and demonstrated a Cohen's Kappa of 0.55 .

A LAA was diagnosed in $37 \%(n=124)$ of all patients. The defects were unilateral in 73 patients (38 on the right and 35 on the left) and bilateral in 51 patients. Patients with avulsion defects were significantly more likely to have an UpfmC on ultrasound; $53.8 \%(n=100)$ when compared with $16.1 \%(n=24)$ in women with a normal contraction $(P<0.001)$. Those with an UpfmC had a higher median defect score than those with $\mathrm{NpfmC}, 8.0$ versus $1.2(P<$ 0.001). All dimensions of the levator hiatus were reduced more effectively in women judged to have $\mathrm{NpfmC}$ as

Table 2 Mean percentage differences and 95\% confidential intervals $(\mathrm{CI})$ of the hiatal dimensions of the levator ani in UpfmC and $\mathrm{NpfmC}$

\begin{tabular}{llll}
\hline Contraction & UpfmC $(n=186)$ & NpfmC $(n=149)$ & $P$ values \\
\hline 2D AP (\% difference, 95\% CI) & $7 \%(-3-16 \%)$ & $18 \%(-3-17 \%)$ & $19 \%(7-33 \%)$ \\
3D AP (\% difference, 95\% CI) & $7 \%(-2-19 \%)$ & $10 \%(-6-27 \%)$ & $P<0.001$ \\
3D LR (\% difference, 95\% CI) & $2 \%(-10-15 \%)$ & $25 \%(9-43 \%)$ & $P<0.001$ \\
3D hiatal area (\% difference, $95 \%$ CI) & $7 \%(7-25 \%)$ & $P<0.001$ \\
\hline
\end{tabular}

$2 D A P$ two-dimensional antero-posterior diameter, $3 D A P$ three-dimensional antero -posterior diameter, $3 D L R$ three-dimensional left-right diameter, $3 D$ hiatal area three-dimensional hiatal area diameter (as measured in Figs. 1 and 2) 
Table 3 Patients demographics in women with $\mathrm{UpfmC}$ and $\mathrm{NpfmC}$ as subjectively assessed with ultrasound

\begin{tabular}{lccc}
\hline Contraction & UpfmC & NpfmC & $P$ value \\
\hline Age, years (mean, SD) & $56.1(\mathrm{SD} \pm 15.4)$ & $54.8(\mathrm{SD} \pm 14.3)$ & 0.48 \\
Age delivery, years (mean, SD) & $26.7(\mathrm{SD} \pm 5.5)$ & $25.4(\mathrm{SD} \pm 4.8)$ & 0.06 \\
Maximum birth weight, g (mean, SD) & $3,655(\mathrm{SD} \pm 670)$ & $3,630(\mathrm{SD} \pm 570)$ & 0.83 \\
Instrumental delivery \% (n) & $11.3 \%(21)$ & $12.1 \%(18)$ & 0.83 \\
Vaginal delivery & & & \\
No \% (n) & $10.2 \%(19)$ & $8.7 \%(13)$ & 0.69 \\
Yes \% (n) & $87.1(162)$ & $86.6 \%(129)$ & \\
\hline
\end{tabular}

opposed to the women with an underactive contraction. For the 2D AP dimension a mean reduction of $18 \%$ for $\mathrm{NpfmC}$ as opposed to $7 \%$ for an UpfmC was found. And for the hiatal area, patients with an UpfmC were less likely to reduce hiatal area on pfmC [mean $7 \%$ reduction vs $25 \%$ in NpfmC $(P<0.001)]$ (see Table 2).

No association was found between age, age at first delivery, vaginal delivery, maximum birth weight and delivery mode, and the presence of poor function (see Table 3). In regards to patient symptoms, women complaining of FI were more likely to have UpfmC on ultrasonography $(P<0.01)$. This was not the case for SUI or symptoms of prolapse, clinical diagnosis of the anterior and central compartment, and obstructed defecation (see Table 3). Furthermore, no differences were found for patients with or without any clinical prolapse stage $\geq 2$ (all three compartments; see Table 3). Patients with a posterior compartment prolapse did have a significant better contraction $(P=0.023$; see Table 4$)$.

\section{Discussion}

With transperineal ultrasound, dynamic imaging can be performed to obtain information on PFMF and anatomic abnormalities of the pelvic floor. This study showed that PFMF can be subjectively qualified and quantitatively assessed on pelvic floor ultrasound. Secondarily, we found an association between major morphological abnormalities of the levator ani ("avulsion injury or defects") and underactive pelvic floor muscle contractility.

It has previously been shown that pelvic floor contraction can be quantified by transperineal ultrasound [18-20]. The present study demonstrates that it is also possible to perform qualitative assessment of pelvic floor contraction as being normal or underactive, however, with a moderate repeatability. In women with an $\mathrm{NpfmC}$ the hiatal area was reduced by $25 \%$. This is in concordance with findings reported by Braekken et al. who investigated pelvic floor muscle contraction by 4D ultrasound in 17 volunteers [19]. Women with UpfmC were able to reduce the hiatal area by only $7 \%$. To date, no information has been obtained on objective measurements on ultrasound of quantification of pelvic floor muscle contraction as normal or abnormal in symptomatic or asymptomatic patients. The present study reports the prevalence of poor function of the pelvic floor in a symptomatic population of $56 \%$.

A limitation of the current study is that we did not compare our ultrasound findings to those with digital palpation. However, Dietz et al. [21] earlier demonstrated in a retrospective study on 1,112 women that there is a significant association between avulsion defects and a reduction in overall contractility ability, as evidenced by the Oxford score and assessed by digital palpation.

Underactive pelvic floor contractility coincided with a higher prevalence of major levator defects in $54 \%$ of
Table 4 Patients symptoms and clinical findings of pelvic organ prolapse (POP-Q stage $\geq 2$ ) in women with $\mathrm{UpfmC}$ versus $\mathrm{NpfmC}$

\begin{tabular}{lccc}
\hline Contraction & UpfmC & NpfmC & $P$ value \\
\hline Fecal incontinenc,e \% $(n)$ & $20.4(38)$ & $8.1(12)$ & 0.002 \\
Stress urinary incontinence, \% $(n)$ & $31.2(58)$ & $37.6(56)$ & 0.22 \\
Obstructed defection, \% $(n)$ & $9.7(18)$ & $8.7(13)$ & 0.77 \\
Anterior compartment prolapse \% $(n)$ & $41.9(78)$ & $36.2(53)$ & 0.29 \\
Central compartment prolapse, \% $(n)$ & $18.8(35)$ & $19.5(29)$ & 0.88 \\
Posterior compartment prolapse, \% (n) & $24.2(45)$ & $35.6(53)$ & 0.023 \\
Any prolapse st $\geq 2$ all three compartments) & & & \\
No, \% $(n)$ & $44.6(83)$ & $39.6(59)$ & 0.36 \\
Yes, \% $(n)$ & $55.4(103)$ & $60.8(90)$ & \\
\hline
\end{tabular}


women versus $16 \%$ in women who were able to contract the pelvic floor muscles normally on ultrasonography. This higher prevalence of defects in the group with poorer PFMF is in concordance with a previous study reported by DeLancey et al. [9]. Another limitation of the present study was that we did not perform a test-retest series for levator defects. However, several studies have shown that major levator defects can be determined with good reproducibility and interobserver agreement including the first author and others $[5,22,23]$.

We found a significant association between FI and weak or absent pfmC. Dysfunction of the PFMF in patients with FI has previously been reported by other studies. Fernandez-Fraga et al. [24] reported reduced levator ani contraction, evaluated with a perineal dynamometer, in patients with FI. Bharucha et al. [25] evaluated PFMF with dynamic MRI in 52 patients with idiopathic FI and 21 controls. They found impaired puborectalis function in 56\% of patients with puborectalis atrophy. The present study did not focus on puborectalis atrophy and its relation with PFMF. However, in women complaining of FI poorer PFMF might explain why pelvic floor muscle training has been reported to be less successful [26, 27].

Urinary continence in women is believed to rely on intrinsic urethral function and urethra vaginal support [28, 29]. DeLancey et al. [29] recently claimed that poor intrinsic urethral function is the predominant factor associated with stress incontinence and not the urethral support of the pelvic floor. This hypothesis seems to be supported by findings that morphological abnormalities of the levator ani were not associated with a higher prevalence of SUI [5], and our results that poorer PFMF does not correlate with an increase of symptoms of SUI. This is surprising, considering the initial management of SUI involves PFMF training. However, recently, it has been concluded that the immediate response to pelvic floor exercises is relatively modest $[12,26]$ and that initial success is often not sustained at long term follow-up [30, 31].

Major LAAs are associated with POP of all three compartments, specifically for the anterior and central compartment $[5,9,11]$. This study showed no difference in regards to PFMF for these complaints for the anterior and central compartment and between women with or without any prolapse stage $\geq 2$ and women with or without any prolapse on clinical examination. For the posterior compartment, however, we found significantly more patients with a normal pfmC. As rectocele have been diagnosed in nullipara, asymptomatic women with assumingly normal pfmC [32], we can only hypothesize that the etiology of developing posterior compartment prolapse might be slightly different. It also could be related to non-relaxation of the pelvic floor, such as anismus and has to be investigated further. Analyzing
PFMF with a vaginal speculum recording force of vaginal closure DeLancey [9] reported in 2007 that women with prolapse generated less force than women without prolapse. The difference in results with our findings might be explained by the different methods used for analyzing PFMF as the measuring force for analyzing PFMF is most likely not comparable with measuring PFMF by contraction visualized on ultrasound as reported in this publication. A Cochrane review conducted by Hagen et al. [33] included the results of three randomized trials and concluded that there was no sufficient evidence for assessing the value of conservative treatment in women with POP.

We performed a retrospective analysis in a selected symptomatic patient group, without using healthy controls. But, as our findings have been supported by previous studies, we believe that further studies are warranted to investigate if PFM dysfunction and anatomical abnormalities of the levator ani might influence the results of conservative treatment, specifically in patients complaining of FI.

In conclusion, the present study showed that pelvic floor muscle function can be qualitatively and quantitatively assessed on pelvic floor ultrasound. An UpfmC is associated with an increased prevalence of major abnormalities of the levator ani. There was an association between FI and poorer pelvic floor function. However, for SUI and pelvic organ prolapse of the anterior and central compartment, this association was not depicted.

\section{Conflicts of interest None.}

Open Access This article is distributed under the terms of the Creative Commons Attribution Noncommercial License which permits any noncommercial use, distribution, and reproduction in any medium, provided the original author(s) and source are credited.

\section{References}

1. Bo K, Finckenhagen HB (2001) Vaginal palpation of pelvic floor muscle strength: inter-test reproducibility and comparison between palpation and vaginal squeeze pressure. Acta Obstet Gynecol Scand 80:883-887

2. Messelink B, Benson T, Berghmans B, Bo K, Corcos J, Fowler C et al (2005) Standardization of terminology of pelvic floor muscle function and dysfunction: report from the pelvic floor clinical assessment group of the International Continence Society. Neurourol Urodyn 24:374-380

3. Dietz HP (2004) Levator function before and after childbirth. Aust N Z J Obstet Gynaecol 44:19-23

4. DeLancey JO, Kearney R, Chou Q, Speights S, Binno S (2003) The appearance of levator ani muscle abnormalities in magnetic resonance images after vaginal delivery. Obstet Gynecol 101:46-53 
5. Dietz HP, Steensma AB (2006) The prevalence of major abnormalities of the levator ani in urogynaecological patients. BJOG 113:225-230

6. Steensma AB, Oom DM, Burger CW, Schouten WR (2009) Assessment of posterior compartment prolapse; a comparison of evacuation proctography and $3 \mathrm{D}$ transperineal ultrasound. Colorectal Dis (in press)

7. Bo K, Sherburn M (2005) Evaluation of female pelvic-floor muscle function and strength. Phys Ther 85:269-282

8. Terra MP, Beets-Tan RG, Vervoorn I, Deutekom M, Wasser MN, Witkamp TD et al (2008) Pelvic floor muscle lesions at endoanal MR imaging in female patients with faecal incontinence. Eur Radiol 18:1892-1901

9. DeLancey JO, Morgan DM, Fenner DE, Kearney R, Guire K, Miller JM et al (2007) Comparison of levator ani muscle defects and function in women with and without pelvic organ prolapse. Obstet Gynecol 109:295-302

10. Dietz HP, Lanzarone V (2005) Levator trauma after vaginal delivery. Obstet Gynecol 106:707-712

11. Dietz HP, Simpson JM (2008) Levator trauma is associated with pelvic organ prolapse. BJOG 115:979-984

12. Brostrom S, Lose G (2008) Pelvic floor muscle training in the prevention and treatment of urinary incontinence in womenwhat is the evidence? Acta Obstet Gynecol Scand 87:384-402

13. Hay-Smith EJ, Dumoulin C (2006) Pelvic floor muscle training versus no treatment, or inactive control treatments, for urinary incontinence in women. Cochrane Database Syst Rev doi:10.1002/14651858. CD005654

14. Abrams P, Cardozo L, Fall M, Griffiths D, Rosier P, Ulmsten U et al (2003) The standardisation of terminology in lower urinary tract function: report from the standardisation sub-committee of the International Continence Society. Urology 61:37-49

15. Dietz HP (2004) Ultrasound imaging of the pelvic floor. Part I: two-dimensional aspects. Ultrasound Obstet Gynecol 23:80-92

16. Dietz HP, Shek C, Clarke B (2005) Biometry of the pubovisceral muscle and levator hiatus by three-dimensional pelvic floor ultrasound. Ultrasound Obstet Gynecol 25:580-585

17. Dietz HP (2007) Quantification of major morphological abnormalities of the levator ani. Ultrasound Obstet Gynecol 29:329-334

18. Dietz HP, Wilson PD, Clarke B (2001) The use of perineal ultrasound to quantify levator activity and teach pelvic floor muscle exercises. Int Urogynecol $\mathrm{J}$ and Pelvic Floor Dysfunct 12:166-168, discussion 168-169

19. Braekken IH, Majida M, Ellstrom-Engh M, Dietz HP, Umek W, Bo K (2008) Test-retest and intra-observer repeatability of two-, three- and four-dimensional perineal ultrasound of pelvic floor muscle anatomy and function. Int Urogynecol $\mathrm{J}$ Pelvic Floor Dysfunct 19:227-235
20. Thyer I, Shek C, Dietz HP (2008) New imaging method for assessing pelvic floor biomechanics. Ultrasound Obstet Gynecol 31:201-205

21. Dietz HP, Shek C (2008) Levator avulsion and grading of pelvic floor muscle strength. Int Urogynecol J Pelvic Floor Dysfunct 19:633-636

22. Weinstein MM, Jung SA, Pretorius DH, Nager CW, den Boer DJ, Mittal RK (2007) The reliability of puborectalis muscle measurements with 3-dimensional ultrasound imaging. Am J Obstet Gynecol 197(68):e61-e66

23. Morgan DM, Umek W, Stein T, Hsu Y, Guire K, DeLancey JO (2007) Interrater reliability of assessing levator ani muscle defects with magnetic resonance images. Int Urogynecol J Pelvic Floor Dysfunct 18:773-778

24. Fernandez-Fraga X, Azpiroz F, Malagelada JR (2002) Significance of pelvic floor muscles in anal incontinence. Gastroenterology 123:1441-1450

25. Bharucha AE, Fletcher JG, Harper CM, Hough D, Daube JR, Stevens C et al (2005) Relationship between symptoms and disordered continence mechanisms in women with idiopathic faecal incontinence. Gut 54:546-555

26. Hay-Smith J, Morkved S, Fairbrother KA, Herbison GP (2008) Pelvic floor muscle training for prevention and treatment of urinary and faecal incontinence in antenatal and postnatal women. Cochrane Database Syst Rev doi:10.1002/ 14651858.CD007471

27. Norton C, Cody JD, Hosker G (2006) Biofeedback and/or sphincter exercises for the treatment of faecal incontinence in adults. Cochrane Database Syst Rev doi:10.1002/14651858. CD002111.pub2

28. DeLancey JO (1988) Structural aspects of the extrinsic continence mechanism. Obstet Gynecol 72:296-301

29. DeLancey JO, Trowbridge ER, Miller JM, Morgan DM, Guire K, Fenner DE et al (2008) Stress urinary incontinence: relative importance of urethral support and urethral closure pressure. J Urol 179:2286-2290, discussion 2290

30. Glazener CM, Herbison GP, MacArthur C, Grant A, Wilson PD (2005) Randomised controlled trial of conservative management of postnatal urinary and faecal incontinence: six year follow up. BMJ 330:337

31. Bo K, Kvarstein B, Nygaard I (2005) Lower urinary tract symptoms and pelvic floor muscle exercise adherence after 15 years. Obstet Gynecol 105:999-1005

32. Dietz HP, Clarke B (2005) Prevalence of rectocele in young nulliparous women. Aust N Z J Obstetr Gynaecol 45:391-394

33. Hagen S, Stark D, Maher C, Adams E (2006) Conservative management of pelvic organ prolapse in women. Cochrane Database Syst Rev doi:10.1002/14651858.CD003882.pub3 